\title{
Black hole in the West nucleus of Arp 220
}

\author{
D. Downes ${ }^{1}$ and A. Eckart ${ }^{2,3}$ \\ 1 Institut de Radio Astronomie Millimétrique, Domaine Universitaire, 38406 St. Martin d'Hères, France \\ e-mail: downes@iram.fr \\ 2 I.Physikalisches Institut, Universität zu Köln, Zulpicherstrasse 77, 50937 Köln, Germany \\ 3 Max-Planck-Institut für Radioastronomie, Auf dem Hügel 69, 53121 Bonn, Germany
}

Received 14 February 2007 / Accepted 13 March 2007

\begin{abstract}
We present new observations with the IRAM Interferometer, in its longest-baseline configuration, of the $\operatorname{CO}(2-1)$ line and the $1.3 \mathrm{~mm}$ dust radiation from the Arp 220 nuclear region. The dust source in the West nucleus has a size of $0.19^{\prime \prime} \times 0.13^{\prime \prime}$ and a $1.3 \mathrm{~mm}$ brightness temperature of $90 \mathrm{~K}$. This implies that the dust ring in the West nucleus has a high opacity, with $\tau=1$ at $1.1 \mathrm{~mm}$. Not only is the dust ring itself optically thick in the submm and far-IR, but it is surrounded by the previously-known, rapidly rotating molecular disk of size $0.5^{\prime \prime}$ that is also optically thick in the mid-IR. The molecular ring is cooler than the hot dust disk because the $\mathrm{CO}(2-1)$ line is seen in absorption against the dust disk. The dust ring is massive $\left(10^{9} M_{\odot}\right)$, compact (radius $35 \mathrm{pc}$ ), and hot (true dust temperature $170 \mathrm{~K}$ ). It resembles rather strikingly the dust ring detected around the quasar APM 08279+52, and is most unlike the warm, extended dust sources in starburst galaxies. Because there is a strong temperature gradient from the hot dust ring to the cooler molecular disk, the heating must come from a concentrated source, an AGN accretion disk that is completely invisible at optical wavelengths, and heavily obscured in hard X-rays.
\end{abstract}

Key words. galaxies: nuclei - galaxies: kinematics and dynamics - galaxies: ISM - galaxies: individual: Arp 220

\section{Evidence for a black hole, so far}

Evidence is growing for a supermassive black hole in the West nucleus of the Arp 220 merger:

1) X-rays: the Chandra point source in the West nucleus has a 2-10 keV luminosity of $10^{7} L_{\odot}$, or $10^{-5}$ times the FIR luminosity. Extended hard X-ray emission in the vicinity raises the total nuclear X-ray luminosity by an order of magnitude, and one cannot rule out a much greater $\mathrm{X}$-ray flux from an AGN hidden behind $\mathrm{H}_{2}$ column densities $>5 \times 10^{24} \mathrm{~cm}^{-2}$ (Clements et al. 2002; Ptak et al. 2003). Updated astrometry yields an even better positional coincidence of the 3-7 keV peak with the West radio nucleus (Iwasawa et al. 2005). The iron $\mathrm{K} \alpha$ emission at $6.7 \mathrm{keV}$ with a large equivalent width $(1.9 \mathrm{keV})$, found by XMM-Newton, may also indicate a powerful AGN, hidden by high $\mathrm{H}_{2}$ column densities (Iwasawa et al. 2005).

2) Evidence for high column densities that could hide an AGN: numerous radio line interferometer maps show a high gas density toward the West nucleus (e.g., Baan \& Haschick 1995 (BH95); Scoville et al. 1997; Downes \& Solomon 1998 (DS98); Sakamoto et al. 1999; Mundell et al. 2001). High-resolution mid-IR maps from the Keck telescope show a warm dust source in the West nucleus that is opaque at $25 \mu \mathrm{m}$ (Soifer et al. 1999). On a larger spatial scale than the West nucleus, the global Arp 220 continuum spectrum measured by the ISO-LWS indicates a dust $\tau>1$ at
$100 \mu \mathrm{m}$, implying an $\mathrm{H}_{2}$ column density of $>2.7 \times 10^{25} \mathrm{~cm}^{-2}$ (Fischer et al. 1997). The $\mathrm{OH}$ and $\mathrm{H}_{2} \mathrm{O}$ lines observed by ISO-LWS (González-Alfonso et al. 2004), and the strong deficiency in the PAH $7.7 \mu \mathrm{m}$ strength vs. $850 \mu \mathrm{m}$ flux (Haas et al. 2001; Soifer et al. 2002; Spoon et al. 2004) also imply an extinction large enough to hide the hard X-ray emission from an AGN accretion disk.

3) Plausible cm-VLBI candidates for an AGN in the West nucleus are the flat-spectrum sources sources W10, W17, and W42, and the rapidly-varying source W33 (Parra et al. 2007; Lonsdale et al. 2006). VLBA monitoring of the radio supernovae within the Arp 220 nuclei suggest that the supernova rate and starburst efficiency should be revised downward, which would lower the starburst contribution to the total luminosity (Rovilos et al. 2005).

4) Kinematic data: the $\mathrm{CO}$ has a very high velocity spread in the West nucleus. One of the $\mathrm{OH}$ masers has a high velocity gradient possibly marking the site of an AGN (Rovilos et al. 2003). Broad ammonia absorption, with a velocity spread of $700 \mathrm{~km} \mathrm{~s}^{-1}$, suggests molecular material in a small, rapidly rotating disk surrounding a black hole (Takano et al. 2005). While the large-scale (15 to $28 \mathrm{kpc}$ ) ionized gas is dominated by tidal motions rather than galactic winds (Colina et al. 2004), the complex kinematics of the ionized gas in the central $2 \mathrm{kpc}$ is influenced by outflows from the dustenshrouded nucleus - see the $\mathrm{H} \alpha$ and [N II] results (Arribas et al. 2001), and the Chandra data (McDowell et al. 2001). 


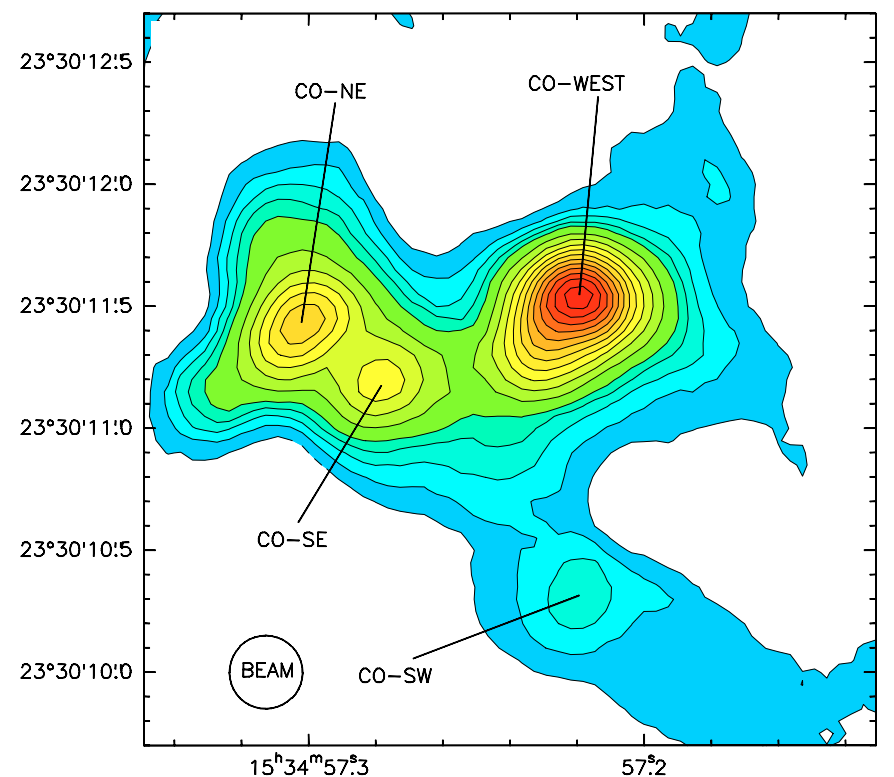

Fig. 1. The central $3^{\prime \prime}$ of Arp 220 in $\mathrm{CO}(2-1)$ integrated over $770 \mathrm{~km} \mathrm{~s}^{-1}$, with the $1.3 \mathrm{~mm}$ continuum subtracted. The beam (lower left ) is $0.30^{\prime \prime}$ with $T_{\mathrm{b}} / S=266 \mathrm{~K} / \mathrm{Jy}$. Contours are 2 to 10 by 2 , then 14 to 54 by 4 (in Jy km s${ }^{-1}$ ). The CO-West peak is $56.5 \mathrm{Jy} \mathrm{km} \mathrm{s}^{-1}$; CO-NE is $33.4 \mathrm{Jy} \mathrm{km} \mathrm{s}^{-1}$.

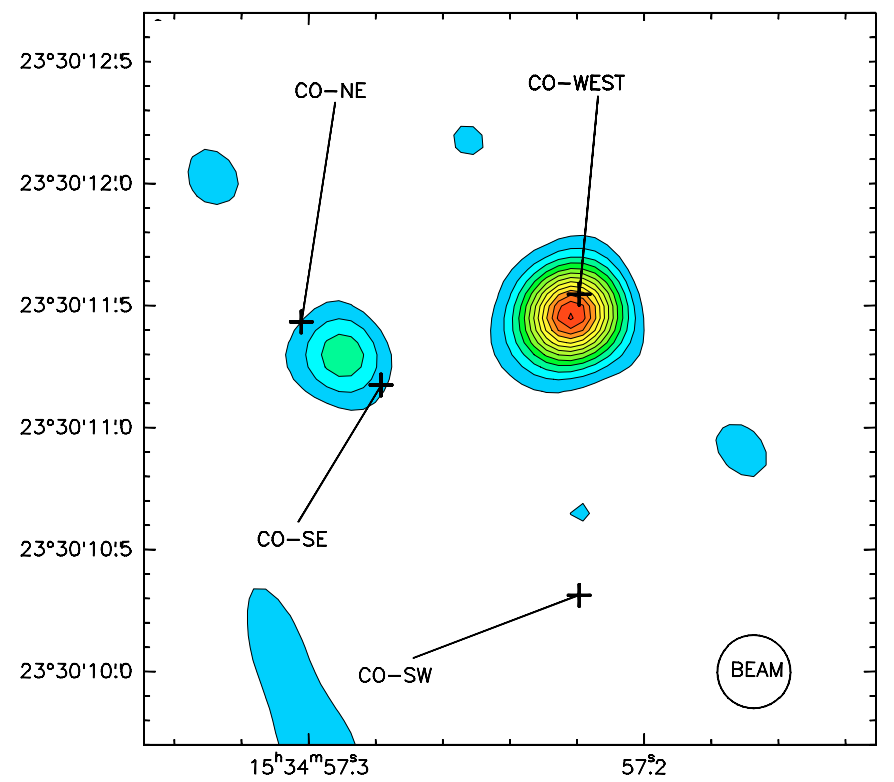

Fig. 2. Continuum map at $1.3 \mathrm{~mm}(229.4 \mathrm{GHz})$. Contour steps are $6 \mathrm{mJy}$ beam $^{-1}$. The Arp 220-West peak is $79 \mathrm{mJy}$ beam $^{-1}$, and the East peak is $23 \mathrm{mJy}_{\text {beam }}{ }^{-1}$. Note that the continuum peaks do not coincide with the $\mathrm{CO}(2-1)$ peaks, which are marked with crosses. The beam is $0.30^{\prime \prime}$ (lower right).

\section{New long-baseline observations}

To further investigate Arp 220's power source, we re-observed the millimeter continuum and the $\mathrm{CO}(2-1)$ and $(1-0)$ lines with the IRAM Plateau de Bure interferometer with its new long baselines to $760 \mathrm{~m}$, which enabled us to restore the data with uniformly-weighted synthesized circular clean beams of $0.30^{\prime \prime}$ at $1.3 \mathrm{~mm}$ and $0.60^{\prime \prime}$ at $2.6 \mathrm{~mm}$. We calibrated amplitudes and phases with 3 C 273, 0923+392, 3C 345, and 1502+106. Receiver temperatures were 45 to $65 \mathrm{~K}$ at both wavelengths.

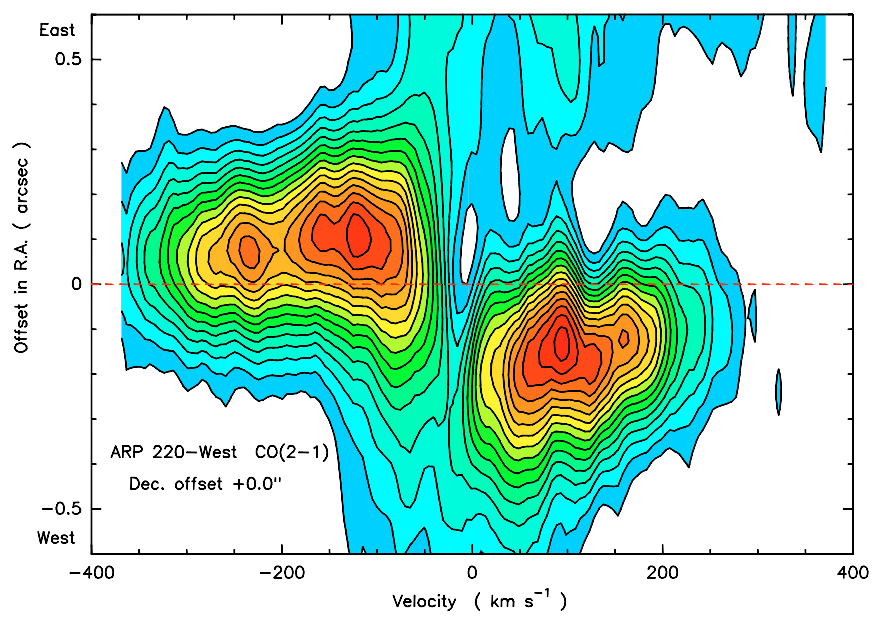

Fig. 3. East-west $\mathrm{CO}(2-1)$ position-velocity cut, through the West nucleus. $\mathrm{CO}(2-1)$ contours are in steps of $10 \mathrm{mJy} \mathrm{beam}^{-1}$, with the $1.3 \mathrm{~mm}$ continuum subtracted. The CO ring around the West nucleus covers velocities from -370 to $+300 \mathrm{~km} \mathrm{~s}^{-1}$, and is cut by absorption at -60 to $+40 \mathrm{~km} \mathrm{~s}^{-1}$. Velocity offsets are relative to $226.422 \mathrm{GHz}\left(c z_{\mathrm{lsr}}=\right.$ $5450 \mathrm{~km} \mathrm{~s}^{-1}$ ). RA offsets are relative to the West continuum peak, indicated by the horizontal line (position in Table 1).

This Letter reports on the $1.3 \mathrm{~mm}$ results on the West nucleus. We observed the $1.3 \mathrm{~mm}$ continuum in the receivers' upper sideband simultaneously with $\mathrm{CO}(2-1)$ in the lower sideband. The spectral correlators covered $770 \mathrm{~km} \mathrm{~s}^{-1}$ at $1.3 \mathrm{~mm}$, with a channel spacing of $1.66 \mathrm{~km} \mathrm{~s}^{-1}$. Our velocity scales are relative to $226.422 \mathrm{GHz}$, which is the rest frequency of $\mathrm{CO}(2-1)$ divided by $1+z_{\mathrm{lsr}}$, where we took $c z_{\mathrm{lsr}}=5450 \mathrm{~km} \mathrm{~s}^{-1}$ as the cosmological redshift. Toward Arp 220, $V_{\mathrm{lsr}}=V_{\text {hel }}+16.6 \mathrm{~km} \mathrm{~s}^{-1}$, so zero velocity offset on our spectra is $5450 \mathrm{~km} \mathrm{~s}^{-1}$ (LSR) and $5433 \mathrm{~km} \mathrm{~s}^{-1}$ (heliocentric).

The new $\mathrm{CO}(2-1)$ map (Fig. 1) shows four peaks, plus an extension of the CO-West gas to the southeast ("source C" on the maps by BH95). The $1.3 \mathrm{~mm}$ continuum map (Fig. 2) yields a source position (Table 1) that agrees within the errors with our earlier IRAM data (DS98). But an important new result is the small measured size of the West continuum, which is $0.19^{\prime \prime} \times 0.13^{\prime \prime}$. This means that the continuum, which at $1.3 \mathrm{~mm}$ is mainly dust emission, does not trace the same matter as the $\mathrm{CO}(2-1)$. The dust is more compact than the $\mathrm{CO}$ emission, which appears to be in a larger ring or disk around the compact dust core. This is clearly shown in the east-west positionvelocity cut through the West nucleus (Fig. 3), which covers the full velocity range of $700 \mathrm{~km} \mathrm{~s}^{-1}$ in the $\mathrm{CO}$, but with a dramatic change from negative to positive velocities over the central $0.2^{\prime \prime}$, where the $\mathrm{CO}$ contours are cut by a deep absorption trough. The CO absorption is even more spectacular in the individual spectra in steps of $0.1^{\prime \prime}$ across the West nucleus (Fig. 4). The main absorption appears to be $100 \mathrm{~km} \mathrm{~s}^{-1}$ wide and centered on $-10 \mathrm{~km} \mathrm{~s}^{-1}$, with some of the spectra showing a second absorption feature at $+130 \mathrm{~km} \mathrm{~s}^{-1}$. Modeling suggests this is partly absorption of the hotter continuum, and CO self-absorption, implying that the $\mathrm{CO}$-West disk itself has a temperature gradient increasing inwards. This is the first time that this Arp 220-West absorption has been seen in $\mathrm{CO}$, and is mainly due to our improved spatial resolution. In previous larger-beam observations, the $\mathrm{CO}$ absorption has probably been masked by $\mathrm{CO}$ emission in the beam. The $\mathrm{CO}$ absorption probably also explains why the West continuum peak is at a slightly different position than the CO (Fig. 2 and Table 1). 
Table 1. Positions, sizes, and fluxes of the Arp 220-West nuclear disks.

\begin{tabular}{|c|c|c|c|c|c|c|c|c|c|}
\hline \multirow[b]{2}{*}{ Data } & \multirow{2}{*}{$\begin{array}{l}\text { RA } \\
15^{\mathrm{h}} 34^{\mathrm{m}} \\
(\mathrm{J} 2000)\end{array}$} & \multirow{2}{*}{$\begin{array}{l}\text { Dec } \\
23^{\circ} 30^{\prime} \\
(\mathrm{J} 2000)\end{array}$} & \multirow{2}{*}{$\begin{array}{l}\text { Major } \\
\text { axis } \\
\text { (arcsec) }\end{array}$} & \multirow{2}{*}{$\begin{array}{l}\text { Minor } \\
\text { axis } \\
(\operatorname{arcsec})\end{array}$} & \multirow{2}{*}{$\begin{array}{c}\text { PA } \\
\text { (deg) }\end{array}$} & \multirow{2}{*}{$\begin{array}{c}\text { Observed } \\
\text { flux density } \\
(\mathrm{mJy})\end{array}$} & \multicolumn{3}{|c|}{ Model components } \\
\hline & & & & & & & $\begin{array}{l}\text { Dust } \\
\text { (mJy) }\end{array}$ & $\begin{array}{c}\mathrm{H} \text { II } \\
(\mathrm{mJy})\end{array}$ & $\begin{array}{c}\text { non-th. } \\
\text { (mJy) }\end{array}$ \\
\hline \multicolumn{10}{|c|}{ Arp 220-West Continuum: } \\
\hline West $1.3 \mathrm{~mm}$ & $57^{\mathrm{s}} .2226$ & $11.46^{\prime \prime}$ & $0.19^{\prime \prime} \pm 0.01^{\prime \prime}$ & $0.13^{\prime \prime} \pm 0.02^{\prime \prime}$ & $-37^{\circ} \pm 6^{\circ}$ & $106 \pm 2$ & 94 & 10 & 2.7 \\
\hline West $2.6 \mathrm{~mm}$ & $57^{\mathrm{s}} .2221$ & $11.48^{\prime \prime}$ & $0.14^{\prime \prime} \pm 0.08^{\prime \prime}$ & - & - & $25 \pm 1$ & 8.5 & 11 & 4.7 \\
\hline \multicolumn{10}{|c|}{ West-CO(2-1) -380 to $-70 \mathrm{~km} \mathrm{~s}^{-1}$ : } \\
\hline & $57^{s} .2265$ & $11.48^{\prime \prime}$ & $0.37^{\prime \prime} \pm 0.1^{\prime \prime}$ & $0.39^{\prime \prime} \pm 0.1^{\prime \prime}$ & $-32^{\circ} \pm 5^{\circ}$ & 74.2 & - & - & - \\
\hline \multicolumn{10}{|c|}{ West-CO $(2-1)+40$ to $+380 \mathrm{~km} \mathrm{~s}^{-1}$ : } \\
\hline & $57^{\mathrm{s}} .2157$ & $11.51^{\prime \prime}$ & $0.32^{\prime \prime} \pm 0.1^{\prime \prime}$ & $0.22^{\prime \prime} \pm 0.1^{\prime \prime}$ & $-80^{\circ} \pm 5^{\circ}$ & 49.3 & - & - & - \\
\hline
\end{tabular}

Estimated position errors are $\pm 0.001^{\mathrm{s}}$ in RA and $\pm 0.05^{\prime \prime}$ in Dec.

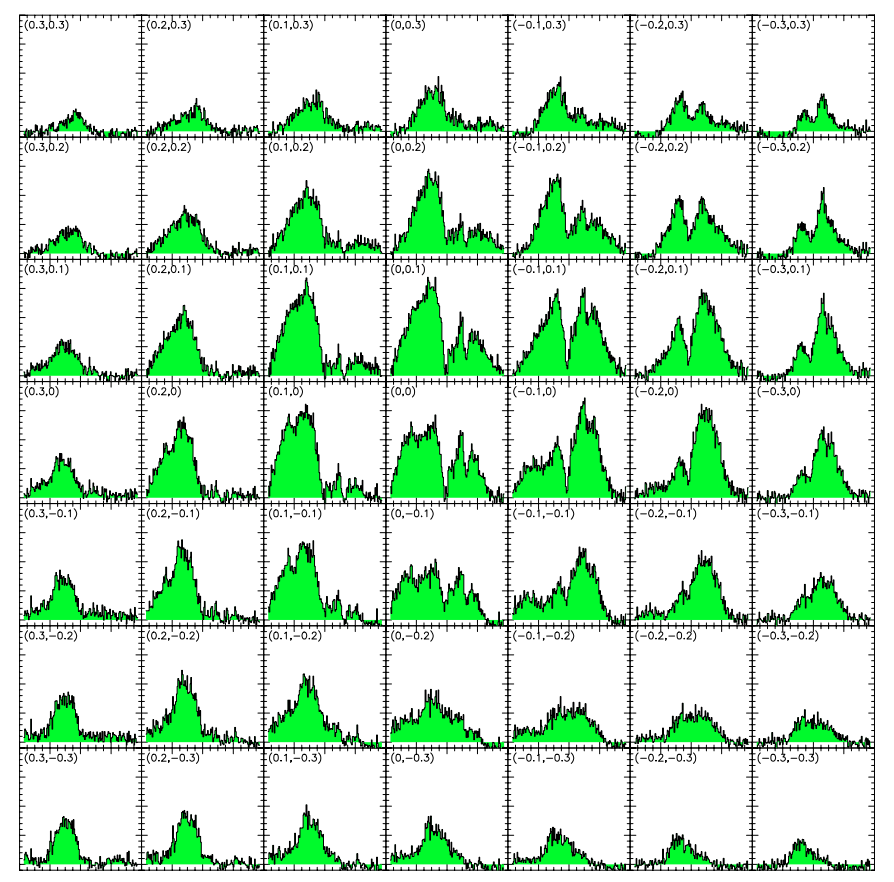

Fig. 4. Evidence for foreground obscuration: $\mathrm{CO}(2-1)$ spectra across Arp 220-West, with the $1.3 \mathrm{~mm}$ continuum subtracted. Each box is a step of $0.1^{\prime \prime}$, north is up, east is left, and (RA, Dec) offsets are in the upper left of each box. The grid center at $(0,0)$ is the $1.3 \mathrm{~mm}$ West continuum peak (Table 1). Velocities run from -400 to $+400 \mathrm{~km} \mathrm{~s}^{-1}$, the spectral resolution is $5 \mathrm{~km} \mathrm{~s}^{-1}$, and zero velocity (the center of the spectra) is at $226.422 \mathrm{GHz}\left(c z_{\mathrm{lsr}}=5450 \mathrm{~km} \mathrm{~s}^{-1}\right)$. Intensities run from 0 to $200 \mathrm{mJy}$ beam $^{-1}$. The beam is $0.30^{\prime \prime}$ with $T_{\mathrm{b}} / S=266 \mathrm{~K} / \mathrm{Jy}$, which means the directly-observed, beam-smoothed, peak CO brightness temperatures are $40 \mathrm{~K}$. Note the deep absorption near the line center in some of the spectra, which indicates foreground, cooler gas.

\section{West dust disk: compact, hot and opaque}

The West continuum peak is $79 \mathrm{mJy}$ in our $0.3^{\prime \prime}$ beam, and its spatially integrated flux is $106 \mathrm{mJy}$, as in our earlier IRAM result (DS98). From lower-frequency data (Sopp \& Alexander 1991; Anantharamaiah et al. 2000; Rodriguez-Rico et al. 2005), we estimate that at $1.3 \mathrm{~mm}$, the West nucleus has an extended synchrotron flux of $2.7 \mathrm{mJy}$, and a free-free continuum of $10 \mathrm{mJy}$ (Table 1; Fig. 5). Extrapolating the $3.6 \mathrm{~cm}$ flux of $400 \mu \mathrm{Jy}$ of the variable VLBI source W33 (Parra et al. 2007) with an assumed spectral index of +0.3 , as in $\mathrm{Sgr} \mathrm{A}^{*}$, we estimate that any synchrotron self-Compton contribution is $<2 \mathrm{mJy}$ at $1.3 \mathrm{~mm}$. Most of the $1.3 \mathrm{~mm}$ flux must therefore be dust emission, at a level of $\sim 94 \mathrm{mJy}$. Hence, the directly-observed, beam-smoothed, dust brightness temperature is $18 \mathrm{~K}$, a remarkably high value at
$1.3 \mathrm{~mm}$. Our Gaussian fits to the West continuum in the $(u, v)$ plane yield a size of $0.19^{\prime \prime} \times 0.13^{\prime \prime}$, so the deconvolved dust brightness temperature is a spectacular $90 \mathrm{~K}$ at $1.3 \mathrm{~mm}$. The Arp 220 west nucleus is thus a very unusual dust source, not at all like the cooler dust sources detected at millimeter wavelengths in starburst galaxies.

The small source size at $1.3 \mathrm{~mm}$ and the deconvolved dust brightness temperature already imply that the $1.3 \mathrm{~mm}$ dust opacity is unusually high. In the simplest estimate, $\tau=-\ln \left(1-T_{\mathrm{b}} / T_{\mathrm{d}}\right)$. If we assume that the intrinsic dust temperature is in the range $\left(90<T_{\mathrm{d}} \leq 180 \mathrm{~K}\right)$, so as not to exceed the Arp 220 IRAS FIR luminosity, then $\tau(1.3 \mathrm{~mm}) \geq 0.7$. This lower limit is already quite a "high" opacity at $1.3 \mathrm{~mm}$. Such a high-brightness dust source at $1.3 \mathrm{~mm}$ will certainly be opaque in the far-IR, so its intrinsic far-IR SED is simply a Planck curve. The spectrum deviates from a blackbody curve on the Wien side however, because it is attenuated by foreground dust. We observe deep $\mathrm{CO}(2-1)$ absorption due to foreground material, so this foreground attentuation must exist. The foreground dust is not as dense as the West-nucleus dust itself; it is optically thin at millimeter wavelengths, but optically thick in the mid-IR. Hence to fit the observed fluxes from the submillimeter through the mid-IR with a blackbody curve, we must first de-redden the observed mid-IR fluxes measured at the Keck telescope by Soifer et al. (1999). In a first iteration, we tried to match the luminosity of the West nucleus derived independently by Soifer et al. and Haas et al. (2001), and started with the foreground dust opacity ( $\tau=1.2$ to 1.5 ) at $25 \mu \mathrm{m}$ that Soifer et al. estimated by $a s-$ suming the source size and dust temperature to be in the range from $\left(0.39^{\prime \prime}, 102 \mathrm{~K}\right)$ to $\left(0.25^{\prime \prime}, 128 \mathrm{~K}\right)$. The source size that we measure, however, is even smaller, $0.19^{\prime \prime} \times 0.13^{\prime \prime}$, implying a higher intrinsic dust temperature to reach the same luminosity, and hence a slightly higher foreground opacity to fit the midIR data points. Our current best compromise is shown in Fig. 5. This solution is for an intrinsic dust temperature of $170 \mathrm{~K}$, a foreground dust opacity of $\tau=1.7$ at $25 \mu \mathrm{m}$, with a $\lambda^{-1}$ opacity dependence at shorter wavelengths, and a $\lambda^{-2}$ dependence at longer wavelengths.

In this solution, the Arp 220-West dust that we observe at millimeter wavelengths (not the foreground dust) has an optical depth of unity at $1.1 \mathrm{~mm}$. For the observed source size, and an intrinsic dust temperature of $170 \mathrm{~K}$, we obtain a total IR luminosity of $9 \times 10^{11} L_{\odot}$ for the West nucleus. The dust flux implies a a gas mass $\left(\mathrm{H}_{2}+\mathrm{He}\right)$ of $1 \times 10^{9} M_{\odot}$, and a mean $\mathrm{H}_{2}$ density of $9 \times 10^{4} \mathrm{~cm}^{-3}$, or $5000 M_{\odot} \mathrm{pc}^{-3}$. The mass could be lower if the dust grains are unusually large, and/or the abundances are supersolar. Such an enclosed mass yields, at radius $30 \mathrm{pc}$, a rotation velocity $(G M / R)^{0.5}$ of $370 \mathrm{~km} \mathrm{~s}^{-1}$, and this is about what we observe in CO. For this West dust source (not the foreground dust), 


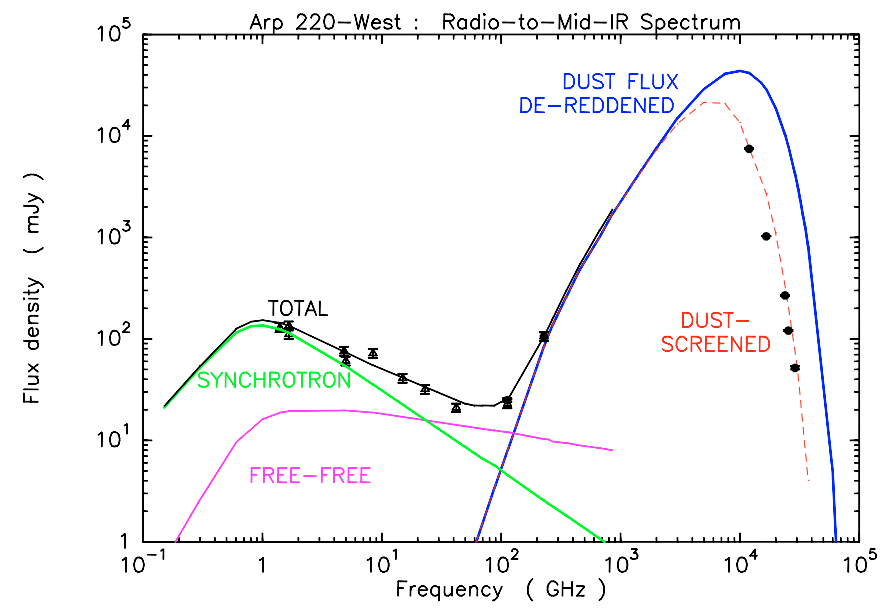

Fig. 5. Our model of the radio-to-mid-IR continuum spectrum of Arp 220-West. The spectrum is dominated by an opaque, $170 \mathrm{~K}$ blackbody dust component, with a luminosity of $9 \times 10^{11} L_{\odot}$, that only becomes optically thin below $1.1 \mathrm{~mm}$. The dust blackbody is absorbed by a foreground screen (the West-CO ring and possibly part of the Eastern disk) that has a foreground opacity of 1.7 at $25 \mu \mathrm{m}$. The effect of the foreground screen is to attenuate the intrinsic mid-IR fluxes, thus shifting the apparent peak of the dust blackbody to longer wavelengths, giving the illusion of a lower temperature (dashed curve). Besides the millimeter-to-mid-IR dust component, there is a free-free component that becomes optically thick below $20 \mathrm{~cm}$, and extended synchrotron emission mixed with the ionized gas, that turns over below $20 \mathrm{~cm}$ due to free-free absorption. The data points at 113 and $229 \mathrm{GHz}$ are from this paper. The other radio fluxes are from the references cited in the text, and the mid-IR points are the 10 to $25 \mu \mathrm{m}$ fluxes measured by Soifer et al. (1999).

if the optical depth scales as $\lambda^{-2}$ to the mid-IR, then we expect $\tau=2000$ at $25 \mu \mathrm{m}$. If $\tau \sim \lambda^{-1}$ from the mid-IR to the visible, then we expect $\tau \approx 10^{5}$ at $5000 \AA$.

What about the foreground dust? The observed CO absorption shows that the West dust core is also obscured by its surrounding, rapidly rotating West molecular disk, and possibly by some of the off-plane, larger-scale, Eastern disk material that may be in front of the West nucleus (see sketch by Mundell et al. 2001). This Eastern disk gas, that we modeled as a warped disk with a quasi polar-ring structure (Eckart \& Downes 2001), is resolved, and therefore mostly absent in the long-baseline data presented here, but it may contribute to the absorption features that cut into the $\mathrm{CO}(2-1)$ line profiles from the West nucleus. For the values in our dust model shown in Fig. 5, the observed flux at $25 \mu \mathrm{m}$ is $7.5 \mathrm{Jy}$ (Soifer et al. 1999). The intrinsic, dereddened dust flux at $25 \mu \mathrm{m}$ is $42 \mathrm{Jy}$. The difference, due the foreground dust of the West molecular disk and possibly part of the Eastern disk, corresponds to a foreground opacity of 1.7 at $25 \mu \mathrm{m}$, close to the value of 1.5 estimated in one of the models by Soifer et al. It also implies a foreground opacity of $\sim 20$ at $K$ band, and a foreground $A_{\mathrm{V}}>100$ in the visible, as previously inferred by Haas et al. (2001) from the weakness of the PAH feature. This foreground extinction is why the Arp 220 has a cooler SED than the "warm", AGN-powered ULIRGs like Mrk 231 and the nearby quasars. At visible wavelengths, the AGN is hidden by $10^{4}$ to $10^{5}$ mag of obscuration by the dust torus, and this dust torus's radiation is itself attenuated by an additional $100 \mathrm{mag}$ due to the compact, $0.5^{\prime \prime}$ West CO disk and possibly part of the larger-scale, warped Eastern CO disk.

\section{Conclusions}

New $\mathrm{CO}(2-1)$ and $1.3 \mathrm{~mm}$ continuum data provide more evidence for a black hole in the Arp 220-West nucleus:

1) The Arp 220-West dust continuum has a $1.3 \mathrm{~mm}$ brightness temperature of $90 \mathrm{~K}$. This is much hotter than the dust detected at millimeter wavelengths from starburst galaxies. SED fitting implies a dust opacity of unity at $1.1 \mathrm{~mm}$, and a true dust temperature of $170 \mathrm{~K}$, so the West disk strongly resembles that of the compact dust toroid around the quasar APM 08279+52 (Egami et al. 2000; Weiss et al. 2007).

2) The size of the West dust source is $35 \times 20 \mathrm{pc}$. This size and the $1.3 \mathrm{~mm}$ dust flux imply a gas density $>5000 M_{\odot} \mathrm{pc}^{3}$, about 10 times the stellar density in cores of giant ellipticals. Model SED fits that attempt to correct upward the mid-IR fluxes for attenuation by the foreground absorbing screen are consistent with a bolometric luminosity of $9 \times 10^{11} L_{\odot}$, that is, $75 \%$ of the total IRAS luminosity of Arp 220. Because of the foreground screen, the true bolometric luminosity is unknown; depending on geometry, it may be significantly higher than the IR luminosity derived from the IRAS data.

3) Strong $\mathrm{CO}$ absorption is seen in front of the dust continuum source. The inner dust source is a hot region, and is not the same source as the surrounding, cooler CO.

4) The West-CO torus centered on the compact $1.3 \mathrm{~mm}$ dust source has a steep velocity rise toward the nucleus, which is characteristic of a massive black hole (Fig. 3). The West molecular gas does not follow a rotation curve rising with radius, typical of inner-galaxy bulge regions. The $\mathrm{CO}$ must be rotating in the gravitational potential of a centrallyconcentrated mass. The $\mathrm{CO}$ velocities in the West molecular torus extend to $370 \mathrm{~km} \mathrm{~s}^{-1}$ at a radius of $30 \mathrm{pc}$, which argues for an enclosed mass (gas + stars + black hole) of $1 \times 10^{9} M_{\odot}$.

5) The data are consistent with the $\mathrm{CO}$ being in a cooler $(50 \mathrm{~K})$ ring surrounding a much hotter $(170 \mathrm{~K})$, dense dust source. The hot dust source is an inner, probably self-gravitating disk of radius $35 \mathrm{pc}$. The radio supernova candidates seen in VLBI maps extend over a slightly larger region, and some of them may be in the West CO torus rather than in the inner, opaque dust disk.

6) The combined proton column densities from the foreground main Arp $220 \mathrm{CO}$ disk $\left(10^{3} \mathrm{~cm}^{-3} \times 300 \mathrm{pc}\right)$, plus the dense CO-West torus $\left(10^{4} \mathrm{~cm}^{-3} \times 90 \mathrm{pc}\right)$, plus the very dense, innermost dust disk $\left(10^{5} \mathrm{~cm}^{-3} \times 30 \mathrm{pc}\right)$ add up to $\sim 1.3 \times$ $10^{25} \mathrm{~cm}^{-3}$. This is sufficient to hide all of the optical emission and most of the hard X-ray emission from the supermassive black hole accretion disk, which must be at the center of the $0.19^{\prime \prime}$ continuum source seen at $1.3 \mathrm{~mm}$.

7) Why it is a black hole: the dust source seen in these millimeter interferometer observations is small $\left(0.19^{\prime \prime} \times 0.13^{\prime \prime}\right)$ and optically thick at $1.1 \mathrm{~mm}$. Its blackbody luminosity is nearly $10^{12} L_{\odot}$. The area of the dust disk on the sky is $2 \times 10^{3} \mathrm{pc}^{2}$, so the emission surface brightness is $\sim 5 \times 10^{14} L_{\odot} \mathrm{kpc}^{-2}$. This puts impossible requirements on a starburst: the equivalent of 10 million $\mathrm{O}$ stars packed into the $r=35 \mathrm{pc}$ dust disk, with $\sim 400$ O stars in each cubic parsec, or 30 times the luminosity of M 82 from a thousand times smaller volume. No such super-starburst has ever been observed. This means the true source of the Arp 220-West luminosity cannot be a starburst. It can only be a black hole accretion disk.

Acknowledgements. We thank the Plateau de Bure Interferometer operators for their help with the observing, C. Thum for useful discussions, and the referee for very helpful suggestions on improving the paper. IRAM is supported by INSU/CNRS (France), MPG (Germany) and IGN (Spain). 


\section{References}

Anantharamaiah, K. R., Viallefond, F., Mohan, N. R., Goss, W. M., \& Zhao, J. H. $2000,537,613$

Arribas, S., Colina, L., \& Clements, D. 2001, ApJ, 560, 160

Baan, W. \& Haschick, A. 1995, ApJ, 454, 745 (BH95)

Clements, D. L., McDowell, J. C., Shaked, S., et al. 2002, ApJ, 581, 974

Colina, L., Arribas, S., \& Clements, D. 2004, ApJ, 602, 181

Downes, D., \& Solomon, P. M. 1998, ApJ, 507, 615 (DS98)

Eckart, A., \& Downes, D. 2001, ApJ, 551, 730

Egami, E., Neugebauer, G., Soifer, B. T., \& Matthews, K. 2000, ApJ, 535, 561

Fischer, J., Satyapal, S., Luhman, M. L., et al. 1997, ESA SP-419, ed. A. Heras et al., ESA, Noordwijk, 149

González-Alfonso, E., Smith, H. A., Fischer, J., \& Cernicharo, J. 2004, ApJ, 613, 247

Haas, M., Klaas, U., Müller, S. A. H., Chini, R., \& Coulson, I. 2001, A\&A, 367, L9

Iwasawa, K., Sanders, D. B., Evans, A. S., et al. 2005, MNRAS, 357, 565

Lonsdale, C. J., Diamond, P. J., Thrall, H., Smith, H. E., \& Lonsdale, C. J. 2006, ApJ, 647, 185

McDowell, J. C., Clements, D. L., Lamb, S. A., et al. 2003, ApJ, 591, 154
Mundell, C. G., Ferruit, P., \& Pedlar, A. 2001, ApJ, 560, 168

Parra, R., Conway, J. E., Diamond, P. J., et al. 2007, ApJ, in press [arXiv:astro-ph/0612248]

Ptak, A., Heckman, T., Levenson, N. A., Weaver, K., \& Strickland, D. 2003, ApJ, 592,782

Rodríguez-Rico, C. A., Goss, W. M., Viallefond, F., et al. 2005, ApJ, 633, 198

Rovilos, E., Diamond, P. J., Lonsdale, C. J., Lonsdale, C. J., \& Smith, H. E. 2003, MNRAS, 342, 373

Rovilos, E., Diamond, P. J., Lonsdale, C. J., Smith, H. E., \& Lonsdale, C. J. 2005, MNRAS, 359, 827

Sakamoto, K., Scoville, N. Z., Yun, M. S., et al. 1999 ApJ, 514, 68

Scoville, N. Z., Yun, M. S., \& Bryant, P. M. 1997, ApJ, 484, 702

Soifer, B. T., Neugebauer, G., Matthews, K., et al. 1999, ApJ, 513, 207

Soifer, B. T., Neugebauer, G., Matthews, K., Egami, E., \& Weinberger, A. J. 2002, AJ, 124, 2980

Sopp, H., \& Alexander, P. 1991, MNRAS, 251, 112

Spoon, H. W. W., Moorwood, A. F. M., Lutz, D., et al. 2004, A\&A, 414, 873

Takano, S., Nakanishi, K., Nakai, N., \& Takano, T. 2005, PASJ, 57, L29

Weiss, A., Downes, D., Neri, R., et al. 2007, A\&A, in press [arXiv:astro-ph/0702669] 